\title{
Elevated Levels of BMP6 Impair Neurogenesis in Alzheimer's Disease
}

\author{
Se Hoon Choi ${ }^{1 \star}$ and Yun $\mathrm{Li}^{2 \star}$ \\ ${ }^{1}$ Genetics and Aging Research Unit, Department of Neurology, Massachusetts General Hospital, Harvard Medical School, Charlestown, Massachusetts \\ 02129, and ${ }^{2}$ The Whitehead Institute for Biomedical Research, Cambridge, Massachusetts 02142 \\ Review of Crews et al.
}

Alzheimer's disease $(\mathrm{AD})$ is a progressive neurodegenerative disease characterized by deterioration of cognitive function and severe neurodegeneration. In addition to the occurrence of amyloid plaques composed of $\beta$-amyloid ( $\mathrm{A} \beta$ ) peptides, neurogenesis and neuronal plasticity are markedly altered in the brain.

In adults, neurogenesis occurs in the subventricular zone (SVZ) of lateral ventricles and in the dentate gyrus of the hippocampus. Recent work indicates that adult hippocampal neurogenesis may play a role in normal brain functions, such as memory formation (for review, see Zhao et al., 2008), and accumulating evidence has shown that neurogenesis is impaired in animal models of $\mathrm{AD}$ (for review, see Lazarov and Marr, 2010), although the mechanism by which adult neurogenesis is impaired in $\mathrm{AD}$ remains ill-defined. A recent study by Crews and colleagues (2010) in The Journal of Neuroscience addressed this question. The study focused on bone-morphogenetic proteins (BMPs). BMPs are members of the transforming growth factor- $\beta$ (TGF- $\beta$ ) superfamily and are important regulators of neurogenesis during embryonic development. During early neural patterning, they

\footnotetext{
Received Oct. 5, 2010; revised Nov. 4, 2010; accepted Nov. 4, 2010.

*S.H.C. and Y.L. contributed equally to this work.

Correspondence should be addressed to Se Hoon Choi, Genetics and Aging Research Unit, Department of Neurology, Massachusetts General Hospital, Harvard Medical School, Charlestown, Massachusetts 02129. E-mail:Choi.SeHoon@mgh.harvard.edu.

DOI:10.1523/JNEUROSCI.5226-10.2011

Copyright $\odot 2011$ the authors $\quad 0270-6474 / 11 / 310371-02 \$ 15.00 / 0$
}

are known to inhibit neurogenesis while enhancing astroglial differentiation. In the CNS, it has been shown that BMP can promote the survival and differentiation of neural progenitors in a region-specific manner (for review, see Mehler et al., 1997). The observation that BMPs are expressed in the adult brain suggests that they may be also involved in adult neurogenesis..

Crews et al. (2010) first examined the levels of three BMPs (BMP2, 6, and 7) in the dentate gyrus of human AD patients. mRNA and protein levels of BMP6, but not BMP2 or BMP7, were significantly increased in the $\mathrm{AD}$ brains compared with nondemented controls (Crews et al., 2010, their Fig. 1). Levels of BMP6 increased as $\mathrm{AD}$ progressed. Immunohistochemical analysis confirmed that BMP6-immunoreactive cells were present in the dentate gyrus and that the number of labeled cells was elevated in $\mathrm{AD}$ brains. To ensure that the marked increased BMP6 level contributes to functional perturbation, it would be useful to examine whether the increase in BMP6 level leads to heightened activity of canonical signaling pathways downstream of BMPs, by measuring the level of phosphorylated Smads. Of equal interest is the cell type(s) responsible for the enhanced BMP6 level. BMP6 expression is limited to neurons in normal adult brain; however, reactive astrocytes also express this protein after traumatic brain injury (Zhang et al., 2006). Given the evidence that reactive astrocytes are prominent in $\mathrm{AD}$ and that normal astrocytes play important roles in creating the neurogenic niches of the adult brain, it would be informative to study whether astrocytes also secrete BMP6 in the AD brain.

Next, Crews et al. (2010) used immunostaining for BMP6 and $\mathrm{A} \beta$ to examine whether increased BMP6 levels in AD might be related to $\mathrm{A} \beta$ pathology. BMP6 protein accumulated around $\mathrm{A} \beta$ plaques in the hippocampus and the cortex of $\mathrm{AD}$ patients (Crews et al., 2010, their Fig. 2). A halo-like pattern of BMP6 immunoreactivity was detected surrounding mature plaques, suggesting that accumulation of $\mathrm{A} \beta$ might affect BMP6 expression or distribution.

To examine whether elevated BMP6 levels affect neurogenesis in $\mathrm{AD}$ brain, the authors performed immunohistochemistry with antibodies against the neuroblast marker doublecortin (DCX) and the early neural progenitor cell marker SOX2 in human brains. Quantification of DCXpositive cells and SOX 2 immunoreactivity revealed that the number of newborn cells was significantly reduced in the $\mathrm{AD}$ patients compared with nondemented controls (Crews et al., 2010, their Fig. 3).

The authors conducted similar studies using transgenic mice expressing high levels of human amyloid precursor protein, and found significantly increased BMP6 levels, accumulation of BMP6 surrounding plaques, and reduced neurogenesis in the dentate gyrus (Crews et al., 2010, their Figs. 4, 5). The similarity between $\mathrm{AD}$ patients and transgenic mice should allow investigation of whether elevated levels of BMP6 in AD mice affect cell-fate determi- 
nation of adult-born cells. This is relevant because various animal models of $\mathrm{AD}$ exhibit altered cell-fate determination (for review, see Lazarov and Marr, 2010) and BMP signaling in SVZ precursors has been shown to direct glial differentiation at the expense of neurogenesis (Lim et al., 2000).

In their next experiment, Crews et al. (2010) showed that $\mathrm{A} \beta$ may directly modulate BMP6 expression in vitro. Treating cultured adult rat hippocampal neural progenitor cell (NPC) with recombinant A $\beta 1-42$ significantly increased BMP6 expression was (Crews et al., 2010, their Fig. 6). Multimeric forms of $\mathrm{A} \beta$ (monomers, dimers, and trimers) were present in the cell lysates of NPC exposed to $A \beta$, suggesting that aggregation of $\mathrm{A} \beta$ may play a role in the upregulation of BMP6 expression. To investigate whether BMP6 directly modulates NPC proliferation, the authors treated cultured NPCs with recombinant BMP6 and measured their proliferation. BMP6 significantly reduced NPC proliferation in a dose-dependent manner with no apparent toxicity (Crews et al., 2010, their Fig. 7).

From these results, the Crews et al. (2010) conclude that $A \beta$-associated increase of BMP6 expression in the hippocampus of $\mathrm{AD}$ patients and $\mathrm{AD}$ transgenic mice may play a role in impairing NPC proliferation in the hippocampus.

Understanding adult neurogenesis and its function is critical in $\mathrm{AD}$ research because impaired neurogenesis might be partially responsible for impaired cognitive function. As proposed by Crews and colleagues (2010), normalization of BMP expression in $\mathrm{AD}$ models might then present a novel ther- apeutic approach for $\mathrm{AD}$. It is conceivable that $\mathrm{AD}$ symptoms could be partially ameliorated if hippocampal neurogenesis were augmented. Indeed, a recent study reported that treatment with a proneurogenic reagent reversed cognitive impairment in $\mathrm{AD}$ transgenic mice (Blanchard et al., 2010).

Although evidence supports neurogenesis as target for $\mathrm{AD}$, the success of this strategy in $\mathrm{AD}$ brain might be complicated. First, the contribution of adult neurogenesis to cognitive function in humans is unclear, but is generally considered to be low, especially in the aging brain. Therefore, it seems unlikely that enhancing neurogenesis could reverse the estimated $10-15 \%$ loss of total hippocampal volume observed in human $\mathrm{AD}$ patients. Second, although $\mathrm{AD}$ is accompanied by substantial hippocampal atrophy, this atrophy is not regionally restricted to the dentate granule cell layer where adult neurogenesis occurs. The degeneration of pyramidal neurons in CA1, a region thought to be non-neurogenic, correlates with decreased hippocampal volume and declines in memory function. In addition, the entorhinal cortex and neocortex, which are heavily affected in $\mathrm{AD}$, are also non-neurogenic. Furthermore, our current understating is that adult-generated dentate neurons do not migrate outside of the dentate gyrus. Thus, the degree to which enhancing neurogenesis would benefit $\mathrm{AD}$ is unclear in the absence of correcting pathology in other non-neurogenic regions. Finally, even if adult neurogenesis contributes to cognitive function in normal brain, the $\mathrm{AD}$ brain might constitute a hostile environment for new neurons; it remains unknown whether such neurons would mature normally and become functional.

Despite these unanswered questions, the idea of inducing neurogenesis for the treatment of $\mathrm{AD}$ remains plausible. From a practical point of view, understanding adult neurogenesis will provide important insights into brain plasticity in general, which might allow us to harness this unique form of innate regenerative capacity to tackle a wide variety of neurological disorders, including AD.

\section{References}

Blanchard J, Wanka L, Tung YC, CárdenasAguayo MC, Laferla FM, Iqbal K, GrundkeIqbal I (2010) Pharmacologic reversal of neurogenic and neuroplastic abnormalities and cognitive impairments without affecting $\mathrm{A} \beta$ and tau pathologies in $3 \mathrm{xTg}-\mathrm{AD}$ mice. Acta Neuropathol 120:605-621.

Crews L, Adame A, Patrick C, Delaney A, Pham E, Rockenstein E, Hansen L, Masliah E (2010) Increased BMP6 levels in the brains of Alzheimer's disease patients and APP transgenic mice are accompanied by impaired neurogenesis. J Neurosci 30:12252-12262.

Lazarov O, Marr RA (2010) Neurogenesis and Alzheimer's disease: at the crossroads. Exp Neurol 223:267-281.

Lim DA, Tramontin AD, Trevejo JM, Herrera DG, García-Verdugo JM, Alvarez-Buylla A (2000) Noggin antagonizes BMP signaling to create a niche for adult neurogenesis. Neuron 28:713-726.

Mehler MF, Mabie PC, Zhang D, Kessler JA (1997) Bone morphogenetic proteins in the nervous system. Trends Neurosci 20:309-317.

Zhang Z, Trautmann K, Artelt M, Burnet M, Schluesener HJ (2006) Bone morphogenetic protein-6 is expressed early by activated astrocytes in lesions of rat traumatic brain injury. Neuroscience 138:47-53.

Zhao C, Deng W, Gage FH (2008) Mechanisms and functional implications of adult neurogenesis. Cell 132:645-660. 